\title{
Guest editorial to the special issue: Big data analysis and management: climate economics, meteorological hazards and environmental risk
}

\author{
Zaiwu Gong ${ }^{1} \cdot$ Guo Wei $^{2} \cdot$ Mei Cai ${ }^{1} \cdot$ Zhijie Sasha Dong $^{3}$
}

Received: 4 June 2021 / Accepted: 4 June 2021 / Published online: 14 June 2021

(C) The Author(s), under exclusive licence to Springer Nature B.V. 2021

At present, the danger of human beings facing climate change and major weather-related disasters is greater than ever, and they are more sudden, more uncertain, and more destructive. However, research has not adequately addressed these issues by using state-of-theart investigation methods or implementing up-to-date management strategies. In order to reduce the losses caused by weather-related disasters and to achieve sustainable development goals and broader human development objectives, this special issue has collected research papers about theoretical investigations, practical applications, and empirical studies on climate change and meteorological disaster management (the urgency of technological innovation) from the economic and management science perspectives. From these studies, it is demonstrated that climate and meteorological data contain a wealth of applications and research value, and big data analysis provides a deep technical means for analyzing these data.

The scope of this collection covers:

- Climate change economics and management science,

- Disaster damage assessment,

- Reduction in weather related economic damage,

- Meteorological sensor networks,

Guo Wei

guo.wei@uncp.edu

Zaiwu Gong

zwgong@nuist.edu.cn; zwgong26@163.com

Mei Cai

caimei@nuist.edu.cn

Zhijie Sasha Dong

sasha.dong@txstate.edu

1 School of Management Science and Engineering, Nanjing University of Information Science and Technology, 219 Ningliu Road, Nanjing 210044, Jiangsu, China

2 Department of Mathematics and Computer Science, University of North Carolina at Pembroke, One University Drive, Pembroke, NC 28372, USA

3 Ingram School of Engineering, Texas State University, 601 University Drive, San Marcos, TX 78666, USA 
- Big data and smart weather,

- Meteorological financial engineering,

- Disaster emergency management

A number of contributions from a wide range of scholars and practitioners from various disciplines have been selected including, but not limited to, decision science, economics, management science, operational research, applied meteorology, and applied statistics on a variety of applications. In particular, several studies on methodological cross-innovation, empirical applications in the context of climate change economics, decision methods and techniques, meteorological disaster management and emergency management are included.

\section{Climate change economics and management science}

Praharaj, Chen, Zahura, Behl and Goodall carried out a quantitative study of the transportation impacts of recurring floods using crowdsourced traffic and flood incident data. Agency-provided continuous count station traffic data were supplemented by crowdsourced traffic data from location-based apps in Norfolk, Virginia to assess the impacts.

$\mathrm{Liu}, \mathrm{Li}, \mathrm{Xu}$ and Luo explored some insights into how cities may measure and improve their urban resilience systems. They aggregated city-level economic, social, infrastructural, and environmental indicators of 39 major Chinese cities from 2002 to 2017 and converted them into a multi-dimensional resilience index.

Huang, Peng, Wei and Wan proposed an empirical study for the Yangtze River Urban Agglomeration to investigate ecological security alarms and change rules. Incorporating statistical data from 2005 to 2017, the combined weight method was applied to calculate the weight of ecological safety over ecological security contributions.

Kourat, Smadhi, Mouhouche, Gourari, Amin and Bryant assessed the impact of future climate change on rainfed wheat yield in the semi-arid Eastern High Plains (Setif and Bordj Bou Arreridj (BBA)) in Algeria using AquaCrop model. The EURO-CORDEX climate projections were downscaled using the ICHEC-KNMI model under two Representative Concentration Pathway scenarios.

Maposa, Seimela, Sigauke and Cochran combined the bivariate conditional extremes model and time-varying threshold in bivariate extremal dependence modelling of maximum temperature extremes in the Limpopo province of South Africa. The findings of the study revealed both significant positive and negative extreme dependence in some pairs of meteorological stations.

Xie, Xie, Cheng, Chu and Dooling adopted Monte Carlo techniques, Hausdorff distance and state-of-the-art map tools to explore the changes in cyclone landing areas along the southeast coast of China in the west coast of the Pacific Ocean, using historical data since 1960s.

Cai and Marson proposed a Natech risk assessment model based on a natural-technological-prone (Natech-prone) facility network to clarify specific scenarios and assess Natech risk. A weighted and directed graph was employed to map out the industrial facilities and other necessary information emerging from the display. A Choquet integral was incorporated into the method to deduce the positive and negative synergistic effects in risk assessment. 


\section{Disaster damage assessment}

Chen, Liu, Pei and Deng focused on the effective management of rainstorm risk for reducing regional rainstorm disaster risks and losses. They discussed the influencing factors of urban rainstorm disasters (URSD) risk from four aspects and then constructed the index system of URSD risk assessment covering 16 influencing factors based on the input of the deep belief nets (DBN) model.

$\mathrm{Yu}, \mathrm{Li}, \mathrm{Huo}$ and Ji employed information diffusion theory to analyze the impacts of five frequent natural disasters on selected areas from 2001 to 2006. Three agricultural indicators, affected area, covered area, and demolished area, were adopted to illustrate the economic losses caused by natural disasters.

Liu and Li used the 2008 to 2015 provincial level data on China's 22 industrial subsectors to investigate both the effect of directed technical change on $\mathrm{CO} 2$ emissions and its heterogeneity. They proposed that the government promote labor biased technical change based on the differentiated characteristics of industry, region, and stage.

Wang, Gong, Shi, Hu and Ahmad conducted a meta-analysis for integrated disaster risk management in the changing climate by surveying 488 papers published from 2008 to 2020. Studies focused in five areas: disaster theory; databases; underlying concepts, relationships, determinants and influences of vulnerability, risk, adaptability and resilience; integrated disaster risk; impact analysis of integrated disaster risk assessment and governance.

Guo, Wu, Guo, Tang and Dzandu investigated the impact of major meteorological events on urban air quality in terms of the extent, duration and spatial scope based on the combination of differences-in-differences and propensity score matching. The study shows a significant improvement in air quality has been achieved in recent years.

Xiong, Zou and Yang proposed a nonlinear time lag multivariable gray prediction model based on interval gray numbers. They took the interval gray number sequence as the modeling sequence for the model to simulate and predict smog index data.

$\mathrm{Wu}$, Zhao, Kuai, Guo and Gao created regional hazard maps of Beijing to directly reflect the degree damage caused by rainstorm disasters in the area. The rainstorm death risk index proposed in this paper can be used to reflect the risk of death caused by rainstorms.

$\mathrm{Xu}, \mathrm{Xu}$ and Yang constructed a risk assessment system and evaluation index of drought disasters, which are composed of a drought risk subsystem, drought exposure subsystem, disaster damage sensitivity subsystem and drought resistance subsystem. Based on the gray matter-element analysis method, they also established the agricultural drought risk evaluation model.

Gan, Wang, Wang, Lev, Shen and Jiang studied how to overcome the adverse impacts of storm debris flow by exploring sustainable interactions between disaster, economy, and ecology. They analyzed the coupling coordination based on order preference for the disaster-economy-ecology system through data-driven technology.

\section{Reduce weather-related economic damages}

Dundas and Haefen found that credible empirical estimation of the economic impacts of climate change is dependent on data structure and the functional relationship between weather data and behavioral outcomes. Using participation data from 1.6 million 
households in the United States from 2004 to 2009, they estimated the impact of temperature and precipitation on participation decisions for marine shoreline recreational fishing.

Cheng, Xu and Sanderson utilized statistical data from 285 cities from 2003 to 2016 in China to study the Environmental Kuznets Curve. They adopted a dynamic spatial panel model to analyze the impact of economic growth on haze pollution and found that economic growth and haze pollution is always closely related, presenting an inverted U-shaped relationship.

Marson and Legerton presented a case study on the challenges faced in addressing the double disasters of Hurricane Matthew (2016) and Hurricane Florence (2018) in Robeson County, North Carolina. They offered a theoretical connection between climate disaster and economic dislocation and the necessary challenge of addressing and reconstructing both at the same time.

Zhu, Jin, Managi and Xun studied the economic consequences of existing natural disasters. They applied panel data from 31 provinces and cities in China from 2010 to 2018 as a sample to examine the relationship between meteorological disasters and the unemployment rate and wage levels of urban and rural population and then verified the mediating effect of government relief.

\section{Big data and smart weather}

Wang, Liu and Zhang analyzed diffused tweets and crisis retweeting dynamics amid Covid19 to provide insights into how Twitter was used by the public and how crisis information of tweets was diffused during this crisis. They identified that Twitter was first and foremost used as a news seeking and sharing platform with more than $70 \%$ of the most diffused tweets relating to news and comments on crisis updates.

Tan, Guo, Mohanarajah and Zhou presented a systematic review on how AI models are applied in different natural disaster management (NDM) stages based on 278 studies retrieved from Elsevier Science, Springer LINK and Web of Science.

Xie, Zuo, Xie, Dooling and Mohanarajah applied a machine learning and data-driven approach and utilized multi-featured data from 31 provinces and regions in China to build a Bayesian network (BN) analysis model for predicting air quality index (AQI) and warning of the air pollution risk at a city level.

Sun, Hou and Guo studied the relationship between China's manufacturing industry benefits and air quality, taking into account the role of government policies in the era of big data, as well as the change points in the time series relationship between industry benefits and air quality.

\section{Meteorological financial engineering}

Sheng, Zheng and Zhong applied the propensity score matching method, compared with matched conventional bonds, and found that green bonds are priced at an average negative premium of $7.8 \mathrm{bps}$, implying that green projects may be issued at a lower cost.

$\mathrm{Qu}, \mathrm{Cai}, \mathrm{Xu}$ and Mohamed introduced robust optimization into the entropy minimization model for Single Regional Input-output (SRIO) table estimation. They constructed 
three robust entropy minimization models to construct China's 2016 SRIO tables and subsequently calculate China's embodied CO2 emission.

Marthak, Perez and Mendez-Mediavilla presented a stochastic programming model which considered pre-positioning strategies among food bank facilities located in high risks areas for hurricanes. The model considered the uncertainty associated with the impact of a hurricane at each facility.

\section{Disaster emergency management}

Zheng, Peng, Sheng and Wan considered that the negative effects of haze risk can easily be spread faster and more widely. They employed diffusion rules on cognition from the unfamiliar to the exposed people through an approach of cellular automata. The study of the spread of haze risk information can help increase the public's awareness of responsibility and improve the government's credibility.

$\mathrm{Hu}$, Zhang, Cui, Zhang and Ahmed conducted a survey with 62,903 respondents from China to explore the public's willingness to engage with disaster relief efforts and the degree of participation. They used statistical and correlation analyses to understand the differences and similarities of the public's willingness to take part in disaster relief across gender and age.

Dong, Meng, Christenson and Fulton focused on different natural disasters based on properties such as types, durations, and damages, covering a total of 41,993 tweets. The change of public opinion during different natural disasters and the evolution of people's behavior of using social media for disaster relief are studied.

$\mathrm{Wu}$ and Zhu proposed a new proactive data envelopment analysis (DEA) approach to investigate the impacts of the national carbon emission trading scheme (ETS) on the carbon peak.

In the recent past, we have witnessed a profound de-emphasis in understanding our ecology through the vision of scientific findings. This special issue represents an effort to reveal evidence of environment degradation, to conduct state-of-the-art investigation for predicting future meteorological disasters, to design innovative preventative measures and emergency management strategies to avoid or minimize fatality and property damage, and optimize environmental policies to stop environmental deterioration on the basis of science and technologies.

Publisher's Note Springer Nature remains neutral with regard to jurisdictional claims in published maps and institutional affiliations. 